\title{
Trial Visit Start Rule
}

National Cancer Institute

\section{Source}

National Cancer Institute. Trial Visit Start Rule. NCI Thesaurus. Code C83449.

An axiom that indicates the beginning of a trial visit. 